\title{
Development and Evaluation of the Environment and Communication Assessment Toolkit with Speech-Language Pathologists
}

\author{
Carrie Bruce, M.A., CCC/SLP, ${ }^{1}$ Jennifer A. Brush, M.A., CCC-SLP, ${ }^{1}$ \\ Jon A. Sanford, M.Arch, ${ }^{1}$ and Margaret P. Calkins, Ph.D. ${ }^{1}$
}

\section{ABSTRACT}

Communication dysfunction that results from dementia can be exacerbated by environmental barriers such as inadequate lighting, noisy conditions, poor or absent environmental cues, and visual clutter. Speech-language pathologists (SLPs) should address these environmental barriers as part of a comprehensive treatment plan for clients with dementia. The Environment and Communication Assessment Toolkit for Dementia Care (ECAT) was evaluated by SLPs to determine: (1) changes in awareness of environmental factors prior to and after training; (2) impact of the ECAT on practice as measured by changes in the number of environmental modifications recommended and made prior to and after training; (3) utility of the information as measured by the helpfulness, amount of new information, and usefulness of the ECAT; and (4) usability of the ECAT materials based on ease of use. The SLPs used the ECAT with clients with dementia who had functional limitations and required substantial assistance with daily activities. Results indicate that the ECAT is an effective tool for SLPs, providing information about the impact of the environment on communication and supplying sufficient resources to make recommendations and implement effective interventions. The ECAT successfully increased awareness of environmental modifications, influenced the practice of recommending environmental modifications, and had utility in diverse aspects of clinical practice.

KEYWORDS: Dementia, environmental modifications, communication environment, environmental interventions

${ }^{1}$ I.D.E.A.S., Inc., Kirtland, Ohio.

Address for correspondence: Jennifer A. Brush, M.A., CCC-SLP, I.D.E.A.S., Inc., 8055 Chardon Rd. Kirtland, OH 44094 (e-mail: jbrush@IDEASConsultingInc.com).

Speech Pathologists in Long-Term Care; Guest Editor, Jennifer A. Brush, M.A., CCC-SLP
Semin Speech Lang 2013;34:42-52. Copyright (C) 2013 by Thieme Medical Publishers, Inc., 333 Seventh Avenue, New York, NY 10001, USA. Tel: +1(212) 584-4662. DOI: http://dx.doi.org/10.1055/s-0033-1337394. ISSN 0734-0478. 
Learning Outcomes: As a result of this activity, the reader will be able to (1) describe the impact of the environment on communication of clients with dementia; (2) consider how environmental assessment can be incorporated into existing clinical practices; and (3) identify common environmental modifications that clinicians reported recommending.

Communication disorders associated with dementia can lead to agitation, restlessness, and aggressive or resistive behaviors during assistance with personal care. ${ }^{1-3}$ These disorders often result in dependence or excess disability in a variety of self-care activities that involve communication between partners, including bathing, dressing, and eating. ${ }^{4-6}$ Although it is difficult to estimate the full clinical significance of communication problems between care providers and clients with dementia, improving communication between these two groups offers a very tangible treatment target. ${ }^{7}$

Traditionally, communication, like other abilities, has been linked to individuals' functional deficits. However, research has shown that communication is also impacted by the environment and that modifications to the environment, such as adjusting light and sound levels, can improve communication skills in individuals with dementia. ${ }^{8-14}$

Due to their specialized training as well as their professional scope of practice, speechlanguage pathologists (SLPs) are in a unique position to address the environmental barriers that affect communication in clients with dementia. However, there have been no comprehensive assessment tools or educational resources that enable clinicians to evaluate the communication environment adequately, mitigate the impact of environmental barriers, and facilitate communication through environmental interventions. As a result, the identification of environmental barriers to and facilitators for effective communication is rarely included in treatment plans developed for individuals with dementia. When environmental modifications are considered in treatment plans, they are, as Bourgeois has suggested, often applied to everyone in the same manner, regardless of diagnosis or severity of symptoms. ${ }^{8}$

This article reports on the evaluation of an assessment protocol and resource guide called the Environment and Communication Assessment
Toolkit for Dementia Care (ECAT) that was designed to enable clinicians to identify social and physical environmental barriers and identify environmental interventions that would facilitate communication in individuals with dementia. $^{15}$

\section{IMPORTANCE OF THE ISSUE}

Dementia can affect quality of life, impair level of functioning, reduce social interactions, and possibly result in the expression of behaviors that can disrupt communication between care providers and care recipients. ${ }^{16,17}$ This not only creates stress in care providers but can also negatively affect the efficacy of rehabilitation therapy programs, as clinicians often "give up" doing therapy when they cannot communicate effectively with a client. ${ }^{18,19}$ Moreover, communication deficits can lead to reduced participation in social activities and increased social withdrawal. ${ }^{7}$

Concurrently, care environments that negatively challenge or do not reinforce the remaining skills and abilities of persons with dementia can lead to excess disability and therefore do not support speech therapy goals or facilitate maintenance of skills learned in therapy. ${ }^{20}$ Recent studies suggest that contextual factors play an important role in clients' comfort levels and performance of daily activities and, consequently, are vital to providing effective care for individuals with dementia. ${ }^{13,21}$ For example, van Hoof and colleagues discuss how individuals with dementia may not be able to appropriately communicate their discomfort with environmental stimuli such as air and odors, light and lighting, and noise and room acoustics and suggest the need to optimize these aspects of the environment based on the known age- and dementia-related changes a person may experience. ${ }^{21}$ Moyle and colleagues also reported on the use of environmental modifications to decrease negative stimulation that can impact information processing and 
encourage orientation through improved signs and lighting to assist clients who engage in wandering behaviors. ${ }^{13}$

Clearly, the American Speech-LanguageHearing Association recognizes the importance of social and physical environment factors on communication as evidenced by its adoption of the constructs and language from the World Health Organization's International Classification of Functioning, Disability and Health (ICF) into its Preferred Practice Patterns for the Profession of Speech-Language Pathology and Scope of Practice in Speech-Language Pathology. ${ }^{22-24}$ The ICF is a model of health and activity performance that is based on the interaction between an individual's body structure/functional abilities and the context (including the physical and social environments) in which activity occurs. Although occupational therapists have historically embraced this ecological, person-environment fit approach through a variety of models that identify the complexity of person-environment-occupation interactions, ${ }^{25-28}$ SLPs have limited disciplinespecific models and frameworks that comprehensively consider person-environment interactions.

Despite the lack of person-environment interaction resources geared to the profession, SLPs are held accountable for promoting improved "quality of life by reducing impairments of body functions and structures, activity limitations, participation restrictions, and barriers created by contextual factors." ${ }^{24}$ This suggests that it is necessary for clinicians to understand the relationship between environmental factors and a client's communication abilities as well as identify environmental interventions to improve the client's communication performance. To this end, the ICF clearly provides an important framework for SLPs to represent how a client's functional characteristics, such as vision and hearing, can interact with the physical and social environment to impact how he or she communicates while performing activities of daily living.

\section{ENVIRONMENTAL FACTORS AND COMMUNICATION}

A variety of environmental factors, including the visual and auditory environments, can affect communication between caregivers and residents with dementia. To demonstrate the effects of these factors, several studies have manipulated lighting and contrast, reduced noise, and added communication aids or cues in the long-term care environment to minimize or eliminate barriers and improve communication and functional abilities.

Lighting and enhanced visual contrast can support both communication and the rehabilitation process. Lighting and contrast conditions are important to consider as age- and dementiarelated changes in the eye and visual processing systems, such as sensitivity to glare, acuity reduction, impaired motion and color discrimination, and diminished contrast sensitivity, can have profound negative effects on a client's visual abilities. ${ }^{21,29,30}$ Noell-Waggoner suggests that lighting interventions such as providing indirect lighting sources that can be adjusted, filtering direct light through sheer draperies and shades, and incorporating natural lighting are a central part of the foundation of a supportive environment and a practical intervention to support remaining abilities in longterm care, including supporting communication. ${ }^{31}$ For example, a study to examine the impact of improved lighting and table setting contrast on clients' oral intake during meals found that these modest environmental changes had positive effects not only on caloric intake, but also on the frequency of client-staff conversations, the frequency with which clients started conversations, and the number of questions that clients answered with on-topic responses. $^{32}$

Changes to the auditory environment can also lead to more focused and less stressed interactions, making communication easier between people with dementia and their care providers. The person's ability to use compensatory strategies to overcome normal age-related declines in hearing is negatively influenced by dementia. ${ }^{33}$ Reducing noise through modifying sound levels and introducing better acoustic materials has been shown to enhance sleep, ${ }^{34-36}$ which could reduce daily fatigue and delirium that also impede communication. Other studies have suggested that noise can cause significant stress in people with dementia and that building design should focus on 
reducing distressing noise levels and introducing calming sounds. ${ }^{37-40}$

Supplementing the environment with communication aids or cues has also been shown to increase communication between staff and people with dementia. Several researchers have reported success using verbal announcements and signs, ${ }^{41,42}$ cue cards, ${ }^{43}$ diaries and watches, ${ }^{44}$ and memory aids. ${ }^{45-48}$ Memory books with autobiographical information and daily schedules with prompts have also been reported to increase clients' frequency of utterances, duration of speaking time, and range of discourse characteristics. ${ }^{49}$

\section{LIMITATIONS OF CURRENT SLP ASSESSMENT PRACTICES}

Despite the importance of environment factors, clients' communication performance is often assessed in a clinical setting that does not reflect the environment in which the client typically spends time. In a long-term care setting, for instance, a clinician may take clients to an office to assess speech, language, or cognitive functioning. Unfortunately, the way that a person performs in a therapy office may be quite different than the way she performs in places where there are a greater variety of different contextual factors such as background noise, lighting levels, or visual stimulation. A clinician must understand how important it is to assess both how a client performs in a clinical setting as well as in the day-to-day living setting.

Although typical speech-language pathology communication performance assessments do not include environmental factors, a few exceptions are found within certain specialty areas of speech-language pathology practice such as augmentative and alternative communication. For example, Beukelman and Mirenda developed the Participation Model for assessment that includes social and physical aspects of the environment. ${ }^{50}$ The social environment sections include communication partner skills and knowledge, policy and practice barriers, and attitudinal issues. The physical environment component addresses how adaptations to physical spaces, locations, or structures (e.g., lowering a table to an appropriate height) should be explored during the assess- ment process to determine how communication can be improved. Another example, Blackstone and Hunt Berg's Social Network, examines social environmental aspects such as effective communication strategies for specific communication partners. ${ }^{51}$ Although both of these examples demonstrate concern for the communication environment in making decisions for evaluation and treatment, neither provides sufficient guidance on the specific features (e.g., lighting) of the environment that should be investigated, attributes that should be measured (e.g., light level), or the best treatment interventions for a specific deficit.

\section{AIM OF THE PRESENT STUDY}

The ECAT was developed by a team of SLPs and architectural researchers specializing in environments for people with dementia, with a large contribution from a multidisciplinary advisory panel of experts in communication disorders, dementia, and environmental interventions for people with dementia. The ECAT provides background information about the impact of the environment on communication, an assessment protocol as well as resources that clinicians can use to measure the environment, and a compendium of typical environmental modifications from which clinicians can make informed recommendations for intervention. It is particularly sensitive to the communication needs of older people with speech, language, cognitive, and sensory impairments (because people with dementia typically experience multiple comorbidities) who live in long-term care settings.

The ECAT has three components (the Manual, Assessment Tools, and Intervention Resources), which have been designed for clinicians to evaluate both public (e.g., dining and living rooms) and personal (e.g., resident bedrooms and bathrooms) spaces in the longterm care setting. The manual primarily focuses on providing background information on dementia and the impact of the environment on communication (Introductory Materials). It also includes descriptions of environmental modifications and links them to specific functional limitations that effect communication (Environmental Intervention Strategies). The 
Assessment Tools component contains instructions on evaluating the environment using the provided assessment instruments including questionnaires that guide the clinician through evaluating the environment, sound and light level meters and usage instructions (Using Light and Sound Level Meters), a reading test that helps identify the appropriate text size for the client, a contrast scale to determine the optimal levels for the client, and a compendium of potential environmental modifications to support communication (Assessment Reference). The Intervention Resources component includes details on types of lighting and research about the effects of lighting as well as reproducible communication environment materials such as sequencing cue cards (Sequencing Cards) and signs.

This article reports on the effectiveness of the ECAT in meeting the practice needs of SLPs in long-term care settings for determining environmental barriers to communication among clients with dementia and developing strategies for environmental modifications to facilitate communication. Effectiveness of the ECAT was measured by four factors: awareness, impact, utility, and usability.

\section{METHODS}

This study was conducted over a 12-month period, and data were collected through questionnaires administered at four points in time and treatment plan information on clients collected twice.

- Time 1 (T1) included a pretest focusing on clinicians' general knowledge of environmental modifications and treatment plan information for two clients with dementia with whom the clinicians worked in the previous 2 months.

- Time 2 (T2) was the posttest taken within 1 month of receipt of the ECAT for knowledge gain after reading the ECAT.

- Time 3 (T3) was a survey given after 2 months of using the ECAT with two clients and treatment plan information for two clients with dementia with whom clinicians worked in the previous 2 months.
- Time 4 (T4) was an optional survey given 3 months after the formal study finished to see if and how SLPs were still using the ECAT voluntarily.

Awareness was assessed by changes in knowledge of environmental modifications based on scores between $\mathrm{T} 1$ and $\mathrm{T} 2$, assessed using an online 10 -item test of material in the ECAT. Impact was evaluated by the changes in the number of clinicians making environmental modifications and number of modifications recommended between $\mathrm{T} 1$ and the T3 (2 months after posttraining) and T4 (5 months after posttraining) periods. Utility was assessed at T3 and T4 by SLPs' evaluation of the helpfulness of the materials, usefulness of the information, and the amount of new information that specific parts of the ECAT added to the participant's clinical knowledge. Usability was assessed by ratings of the ease of using the materials at $\mathrm{T} 3$ and $\mathrm{T} 4$.

\section{PARTICIPANTS}

A total of 71 clinicians (i.e., SLPs, nurses, physical and occupational therapists) were recruited to evaluate the ECAT while using it with clients who had a diagnosis of dementia. All of the clinicians reported providing services for clients with dementia who had functional limitations performing activities of daily living. Therapeutic interventions during the study addressed cognition, dysphagia, expressive/receptive communication, fine motor coordination, gross motor coordination, physical aspects of eating, and/or range of motion.

This article focuses on results only from the SLPs. At the pretraining phase (T1), the sample was comprised of 28 SLPs, all of whom were women. More than $96 \%$ of the participants were Caucasian $(n=25)$, with the remaining three clinicians being AfricanAmerican, Hispanic, and American-Indian ethnicities. The participating clinicians ranged in age from 20 to 60 years, although the vast majority were between the ages of 20 and 40 years $(n=20)$. One clinician dropped out before T2 and five clinicians dropped out 
before T3. This left 22 clinicians remaining for the T3 period. At T4, 5 months after posttraining, 18 of the T3 clinicians completed the questionnaire, 16 of whom had voluntarily continued to use the ECAT as part of their clinical practice.

\section{RESULTS}

We were interested in individual SLP performance over time and used paired $t$ tests in the analysis. Therefore, data are only reported for clinicians who completed the relevant assessments for each factor.

\section{Awareness}

Using a paired sample $t$ test, the clinicians who took the pretest had a mean score of $5.19 \%$ on the 10-item knowledge test at T1. At T2, after training with the ECAT materials, the same clinicians $(n=24)$ scored almost 1.6 percentage points higher, a $30 \%$ change in score $(6.77 \% ; p<0.000)$.

\section{Impact}

Impact was assessed in several ways. First, the number of clinicians who made modifications was tracked. Second, the number of modifications they recommended was recorded. Finally, cost of modifications implemented was also tracked.

\section{NUMBER OF CLINICIANS WHO MADE ENVIRONMENTAL MODIFICATION RECOMMENDATIONS}

At T1 baseline, prior to implementing the ECAT, $62 \%$ of the clinicians recommended at least one environmental modification for their clients. At T3, the overall frequency of clinicians who made recommendations increased slightly to $64 \%$. The overall frequency of clinicians making recommendations increased to $89 \%$ at T4.

\section{NUMBER OF ENVIRONMENTAL MODIFICATION RECOMMENDATIONS}

At T1, the clinicians who reported recommending environmental modifications recommended an average of 4.75 modifications for each of their two clients. This increased to 4.8 at $\mathrm{T} 3$ and to 7.35 at T4. Post hoc analyses indicate that there were significant differences between $\mathrm{T} 1$ and T4 $(p=0.001)$ and T3 and T4 $(p=0.007)$, although the difference between $\mathrm{T} 1$ and $\mathrm{T} 3$ was not significant.

The three most common recommendations were: create external memory aids (84.2\%), reduce noise $(82.8 \%)$, and provide signage $(78.3 \%)$. From $\mathrm{T} 1$ to $\mathrm{T} 4$, there were significant increases in several modifications including: maximize visibility of toilet $(p=0.004)$; label items $(p=0.012)$; make bedroom visually distinctive $(p=0.016)$; modify closet $(p=0.028)$; and improve access to TV, radio, or phone $(p=0.038)$. In addition, several modifications that did not reach statistical significance between $\mathrm{T} 1$ and T4 demonstrated a clear trend of approaching significance. These included: increase contrast, which increased from $56.3 \%$ at $\mathrm{T} 1$ to $93.8 \%$ at $\mathrm{T} 4(p=0.052)$; improve way-finding cues, which increased from $50.0 \%$ at $\mathrm{T} 1$ to $81.3 \%$ at T4 $(p=0.055)$; modify lighting controls, which increased from $12.5 \%$ at $\mathrm{T} 1$ to $43.8 \%$ at T4 ( $p=0.064)$; enhance lighting, which increased from $56.3 \%$ at T1 to $87.5 \%$ at T4 $(p=0.070)$; and rearrange furniture, which increased from $43.8 \%$ at $\mathrm{T} 1$ to $75.0 \%$ at T4 $(p=0.070)$.

\section{COST OF ENVIRONMENTAL MODIFICATIONS}

Among those clinicians who responded to questions of cost, there were no significant differences in cost of environmental modifications across T1, T3, and T4. Among the 11 clinicians who reported on cost for their two clients at T1, over one-third (36.4\%) of the clinicians reported that the modifications didn't cost anything, almost half (45.5\%) indicated that modifications cost between $\$ 1$ and $\$ 100$, and the remainder $(9.1 \%)$ reported that the modifications cost between $\$ 101$ and $\$ 250$. When asked about the cost of their most successful environmental modification, all of the clinicians reported that the cost was less than $\$ 100$. At the end of the study (T4), the percentage of clinicians making modifications for less than $\$ 100$ was $87.5 \%$. Again, $100 \%$ reported the cost of their most successful modification at less than $\$ 100$. 


\section{Utility}

\section{HELPFULNESS}

Clinicians were asked to rate the helpfulness of the ECAT during the first 2 months after training (T3) on a 4-point Likert scale. Over half the SLPs (56.4\%) gave the ECAT the highest rating (very helpful), whereas $22.2 \%$ rated it a 2 and $16.8 \%$ rated it as somewhat helpful (score of 3).

\section{NEW INFORMATION}

At T3, the degree of new information included in the different sections of the ECAT was rated on a 4-point scale from 1, "all of the information was new," to 4 , "none of the information was new." Overall, $54 \%$ of the information from different sections was rated as "all" or "most" of it being new, and only $6 \%$ of the sections were rated as having no new information. The SLPs were least familiar with the environmental strategies and sound and light meter use (64\% and $81 \%$ rating these as all or mostly new information, respectively) and were most familiar with sequencing cards (52\% indicating there was some or no new information).

\section{USEFULNESS}

Every clinician indicated at T3 that the ECAT provided new treatment options to use with clients with dementia. The usefulness of the ECAT was further assessed on a 7-point Likert scale on seven questions about future use in practice, information usefulness, practice value, and usefulness in working with clients, care assistants, families, and administration. At T3, the overall mean response to all of the questions was 5.55, and this increased to 6.02 at T4. Some of the strongest responses at $\mathrm{T} 4$ were: it had useful information (90.5\% largely or strongly agreed), added value to their clinical practice (76.2\% largely or strongly agreed), and they will use it in the future $(71.4 \%$ largely or strongly agreed).

\section{Usability}

The usability of the ECAT was measured at T3 on a 7-point Likert scale to assess how easy the information was to understand and use. Twenty of 21 respondents (95.2\%) agreed that the information was easy to understand, with the majority (52.4\%) rating the strength of agreement at 6 on the 7-point scale. Eighteen $(85.7 \%)$ had some level of agreement (rating 5, 6, or 7) with the statement that the ECAT was easy to use, with the majority (42\%) rating the ease of use as 6 . Only one clinician reported that she had some difficulty matching her findings to potential modifications.

\section{DISCUSSION}

This study demonstrated that the ECAT is an effective tool for clinicians in providing information about the impact of the environment on communication and provides resources to make and implement recommendations for interventions. Overall, the ECAT demonstrated success in improving awareness of environmental modifications, affecting the practice of recommending environmental modifications, having utility in diverse aspects of clinical practice, and being usable. The ECAT was also valuable in that it facilitated treatment for clients with dementia who had a range of functional limitations and difficulties with various activities of daily living that required speech-language pathology services.

The most valuable findings were related to the significant increase in the number and type of modifications that clinicians recommended after training with and using the ECAT. On average, the frequency of recommendations rose almost $25 \%$ over the course of the study, indicating that clinicians were recommending more environmental modifications per client at the end of the study compared with the start. A majority of the recommendations made by clinicians focused on supporting clients through modifications that optimize cognitive aspects of the environment such as labeling items, providing time orientation cues, developing sequencing cues, creating external memory aids, and providing signage.

Clinicians overwhelmingly stated that the ECAT added value to clinical practice and would benefit their clients. Additionally, all of the clinicians reported that the ECAT provided more treatment options for their clients and all but one indicated they would continue to use 
the ECAT in the future. Furthermore, the usability of the ECAT was rated positively, with a majority of the clinicians reporting that it was easy to use and the information was easy to understand.

This study had some limitations that should be noted. As is typical in longer-term studies, we had some attrition in participants over time. Despite this decline in subjects, 16 of the 18 SLPs who responded at T4 indicated that they continued to use the ECAT with clients, even though this was not requested as part of the study. Our goal at T4 was to determine if clinicians who had been trained with the ECAT would continue to use it voluntarily. Not only did the majority of T3 SLPs (72\%) continue to use it, but more environmental interventions were being included in treatment plans over time. The sustained interest in using the ECAT indicates that the ECAT is valued among SLPs as a useful clinical treatment resource for working with clients who have dementia.

Another limitation was our lack of comparison to a control group that did not receive the ECAT. Although this was partially addressed through the pre- and postdesign of this study, it would be useful in the future to compare trained and untrained groups over time, particularly to make associations between clients' functional limitations, activity difficulties, and skilled services to recommended environmental modifications. Finally, our data are all based on self-reports from clinicians, which introduces possible problems with inaccurate recall of client or modification details.

The overall goal of speech-language pathology intervention is to optimize an individual's ability to communicate in ecologically valid environments. Therefore, having a focus on an environment that supports an individual's communication needs becomes an important and necessary part of the rehabilitation process. The ECAT both supports systematic and individualized assessment of the environment and provides education and resources on barriers and facilitators to effective communication that will enable clinicians in long-term care settings to minimize or eliminate barriers to communication and introduce communication facilitators.

\section{ACKNOWLEDGMENTS}

This research was supported through NIH grants \#DC008916 awarded to I.D.E.A.S., Inc. We would also like to thank Amanda Kerr and Hannah Fleder for their help in assisting the research team.

\section{REFERENCES}

1. Kolanowski AM, Richards KC, Sullivan SC. Derivation of an intervention for need-driven behavior. Activity preferences of persons with dementia. J Gerontol Nurs 2002;28:12-15

2. Swearer J, Drachman D, O'Donnell B. Troublesome and destructive behaviors in dementias. J Am Geriatr Soc 1998;36:784-790

3. Teri L, Larson EB, Reifler B. Behavior disturbance in dementia of the Alzheimer types. J Am Geriatr Soc 1988;37:109-116

4. Brush JA, Threats TT, Calkins MP. Influences on perceived function of a nursing home resident. J Commun Disord 2003;36:379-393

5. Sloane PD, Hoeffer B, Mitchell CM, et al. Effect of person centered showering and the towel bath on bathing-associated aggression, agitation, and discomfort in nursing home residents with dementia: a randomized, controlled trial. J Am Geriatr Soc 1994;S2:795-804

6. Sloane PD, Honn VJ, Dwyer SAR, Wieselquist J, Cain C, Myers S. Bathing the Alzheimer's patient in long term care: results and recommendations from three studies. Am J Alzheimer Dis 1995;10: 3-12

7. Potkins D, Myint P, Bannister C, et al. Language impairment in dementia: impact on symptoms and care needs in residential homes. Int J Geriatr Psychiatry 2003;18:1002-1006

8. Bourgeois MS. Communication treatment for adults with dementia. J Speech Hear Res 1991; 34:831-844

9. Gottesman LE. Resocialization of the geriatric mental patient. Am J Public Health Nations Health 1965;55:1964-1970

10. Kihlgren M, Bråne G, Karlsson I, Kuremyr D, Leissner P, Norberg A. Long-term influences on demented patients in different caring milieus, a collective living unit and a nursing home: a descriptive study. Dementia 1992;3:342-349

11. McCracken AL, Fitzwater E. The right environment for Alzheimer's: which is better - open versus closed units? Here's how to tailor the answer to the patient. Geriatr Nurs (Minneap) 1989;10:293-294

12. Melin L, Götestam KG. The effects of rearranging ward routines on communication and eating behaviors of psychogeriatric patients. J Appl Behav Anal 1981;14:47-51 
13. Moyle W, Olorenshaw R, Wallis M, Borbasi S. Best practice for the management of older people with dementia in the acute care setting: a review of the literature. Int J Older People Nurs 2008;3: 121-130

14. Skea D, Lindesay J. The evaluation of two models of long-term residential care for elderly people with dementia. Int J Geriatr Psychiatry 1996;11:233241

15. Brush J, Calkins M, Bruce C, Sanford J. Environment and Communication Assessment Toolkit for Dementia Care (ECAT). Baltimore, MD: Health Professions Press; 2012

16. Hopper T. Long-term care residents with dementia: assessment and intervention. The ASHA Leader 2004;24:10-11

17. Le Dorze G, Julien M, Genereux S, LArfeuil C, Nacennec C, LaPorte D, Chanpagne C. The development of a procedure for the evaluation of communication occurring between residents in long-term care and their caregivers. Aphasiology 2000;14:17-51

18. Steeman E, Abraham IL, Godderis J. Risk profiles for institutionalization in a cohort of elderly people with dementia or depression. Arch Psychiatr Nurs 1997;11:295-303

19. Williamson GM, Schulz R. Coping with specific stressors in Alzheimer's disease caregiving. Gerontologist 1993;33:747-755

20. Helen C. Doing lunch: a proposal for a functional well-being assessment. Alzheimer's Care Quarterly 2002;3:302-315

21. van Hoof J, Kort H, Duijnstee M, Rutten P, Hensen J. The indoor environment and the integrated design of homes for older people with dementia. Build Environ 2010;45:1244-1261

22. World Health Organization. International Classification of Functioning, Disability and Health. Geneva, Switzerland2001

23. American Speech-Language-Hearing Association Preferred Practice Patterns for the Profession of Speech-Language Pathology; 2004. Available at: www.asha.org/policy. Accessed on February 26, 2013.

24. American Speech-Language-Hearing Association Scope of Practice in Speech-Language Pathology; 2007. Available at: www.asha.org/policy. Accessed on February 26, 2013.

25. Christiansen C, Baum C. Occupational Therapy: Enabling Function and Well-Being. 2nd ed. Thorofare, NJ: Slack, Inc.; 1997

26. Dunn W, Brown C, McGuigan A. The ecology of human performance: a framework for considering the effect of context. Am J Occup Ther 1994; 48:595-607

27. Hagedorn R. Occupational Therapy: Perspectives and Processes. Edinburgh, Scotland: Churchill Livingstone; 1995
28. Kielhofner G. A Model of Human Occupation: Therapy and Application. 2nd ed. Baltimore, MD: Williams \& Wilkins; 1995

29. Brawley EC. Designing for Alzheimer's Disease. Strategies for Creating Better Care Environments. New York, NY: John Wiley and Sons, Inc.; 1997

30. Kergoat H, Kergoat MJ, Justino L, Robillard A, Bergman H, Chertkow H. Normal optic nerve head topography in the early stages of dementia of the Alzheimer type. Dement Geriatr Cogn Disord 2001;12:359-363

31. Noell-Waggoner E. Light: an essential intervention for Alzheimer's disease. Alzheimer's Care Quarterly 2002;3:343-352

32. Brush J, Meehan R, Calkins M. Using the environment to improve intake for people with dementia. Alzheimer's Care Quarterly 2002;3:330-338

33. Weaverdyck SE. Intervention to address dementia as a cognitive disorder. In: Coon DH, Ed. Specialized Dementia Care Units. Baltimore, MD: John Hopkins University Press; 1991:224-244

34. Berg S. Impact of reduced reverberation time on sound-induced arousals during sleep. Sleep 2001;24:289-292

35. Novaes MA, Aronovich A, Ferraz MB, Knobel E. Stressors in ICU: patients' evaluation. Intensive Care Med 1997;23:1282-1285

36. Southwell MT, Wistow G. Sleep in hospitals at night: are patients' needs being met? J Adv Nurs 1995;21:1101-1109

37. Bakker R. Sensory loss, dementia, and environments. Generations 2003;27:46-51

38. Burgio L, Scilley K, Hardin JM, Hsu C, Yancey J. Environmental "white noise": an intervention for verbally agitated nursing home residents. The Journals of Gerontology: Series B 1996;51B: P364-P373

39. Day K, Carreon D, Stump C. The therapeutic design of environments for people with dementia: a review of the empirical research. Gerontologist 2000;40:397-416

40. Dewing J. Caring for people with dementia: noise and light. Nurs Older People 2009;21:34-38

41. Hanley IG. The use of signposts and active training to modify ward disorientation in elderly patients. J Behav Ther Exp Psychiatry 1981;12:241-247

42. McClannahan LE, Risley TR. Design of living environment for nursing home residents: recruiting attendance at activities. Gerontologist 1974; 14:236-240

43. Smith WL. Behavioral interventions in gerontology: management of behavior problems in individuals with Alzheimer's disease living in the community. In: Association for Behavior Analysis Convention. Philadelphia, PA, 1988

44. Hanley IG, Lusty K. Memory aids in reality orientation: a single-case study. Behav Res Ther 1984;22:709-712 
45. Bourgeois MS. Enhancing conversation skills in patients with Alzheimer's disease using a prosthetic memory aid. J Appl Behav Anal 1990;23:29-42

46. Bourgeois M. Conversing with Memory Impaired Individuals using Memory Aids. Gaylord. MI: Northern Speech Services; 1992

47. Bourgeois MS. Evaluating memory wallets in conversations with persons with dementia. J Speech Hear Res 1992;35:1344-1357

48. Mateer C, Sohlberg M. A paradigm shift in memory rehabilitation. In Whitaker $\mathrm{H}$, Ed. Neuropsychological Studies of Non-focal Brain Injury: Dementia and Closed Head Injury. New York, NY: Springer-Verlag; 1998:202-225
49. Bourgeois M, Dijkstra K, Burgio L, Allen-Burge $\mathrm{R}$. Memory aids as an augmentative and alternative communication strategy for nursing home residents with dementia. Augment Altern Commun 2001; 17:196-210

50. Beukelman D, Mirenda P. Augmentative \& Alternative Communication. 3rd ed. Baltimore, MD: P. H. Brookes; 2005

51. Blackstone SW, Hunt Berg M. Social Networks: An Assessment and Intervention Planning Inventory for Individuals with Complex Communication Needs and Their Communication Partners. Monterey, CA: Augmentative Communication; 2002 